\title{
Domain structure and dielectric properties of metal-ferroelectric superlattices with asymmetric interfaces
}

\author{
Marios Hadjimichael $\odot,{ }^{1, *}$ Yaqi Li, ${ }^{1}$ Lluís Yedra, ${ }^{2,3}$ Brahim Dkhil $\odot,{ }^{2}$ and Pavlo Zubko $\odot^{1}$ \\ ${ }^{1}$ London Centre for Nanotechnology and Department of Physics and Astronomy, University College London, 17-19 Gordon Street, \\ WC1H OHA London, United Kingdom \\ ${ }^{2}$ Laboratoire Structures, Propriétés et Modélisation des Solides, CentraleSupélec, CNRS-UMR8580, Université Paris-Saclay, \\ Gif-sur-Yvette, France \\ ${ }^{3}$ Laboratoire Mécanique des Sols, Structures et Matériaux, CentraleSupélec, CNRS-UMR8579, Université Paris-Saclay, \\ Gif-sur-Yvette, France
}

(Received 4 July 2019; revised 25 July 2020; accepted 24 August 2020; published 29 September 2020)

\begin{abstract}
$\mathrm{PbTiO}_{3} / \mathrm{SrRuO}_{3}$ superlattices deposited on $\mathrm{SrTiO}_{3}$ substrates are studied using a combination of x-ray diffraction, piezoresponse force microscopy, scanning transmission electron microscopy, transport measurements, and impedance spectroscopy. The superlattices are found to have two inequivalent interfaces resulting from differences in the growth modes for $\mathrm{PbTiO}_{3}$ and $\mathrm{SrRuO}_{3}$. X-ray diffraction measurements show that, despite being sandwiched between metallic $\mathrm{SrRuO}_{3}$ layers, the ferroelectric layers possess dense nanoscale domains. The observed domain sizes are comparable to those found in ferroelectric-dielectric systems, and they are attributed to the depolarizing field caused by the finite screening length of the $\mathrm{SrRuO}_{3}-\mathrm{PbTiO}_{3}$ interface. The macroscopic capacitance of the ultrathin $\mathrm{PbTiO}_{3}$ layers was measured, and its temperature dependence was found to be consistent with permittivity enhancement due to domain wall motion.
\end{abstract}

DOI: 10.1103/PhysRevMaterials.4.094415

\section{INTRODUCTION}

Over the past decade, ferroelectric-dielectric superlattices have emerged as a model system for investigating nanoscale ferroelectricity and novel polarization textures. In these artificially layered materials, ordered nanoscale domains form within the ultrathin ferroelectric layers to minimize the depolarization field energy associated with the unscreened polarization [1-3]. Motivated by theoretical predictions of complex polarization structures, unusual switching behavior, and high-frequency dynamics [4-9], the properties of nanodomains in ferroelectric superlattices have been widely investigated using laboratory and synchrotron x-ray diffraction as a function of applied field, temperature, and optical excitation [3,10-21]. Direct imaging of the domains using scanning probe techniques is challenging due to their small sizes and buried nature, and it has been mainly restricted to ferroelectric-dielectric bilayers and trilayers, or superlattices with larger domains [22-24]. Recently, however, the complex polarization textures have been directly observed using transmission electron microscopy, revealing ordered flux-closure domains [25-27], as well as vortexlike and bubblelike structures with gradually curling polarization components and a macroscopic chirality observed by resonant $\mathrm{x}$-ray diffraction [28-31].

Such multidomain superlattices also exhibit unusual dielectric properties, dominated by the highly responsive domain walls. Nanoscale displacements of the densely packed

*marios.hadjimichael@unige.ch domain walls lead to large enhancements in the overall dielectric susceptibility of the superlattices $[3,11]$. Importantly, within the ferroelectric layers, the domain wall response can locally overscreen the applied field, amplifying the potential drop across the adjacent dielectric layers and giving rise to so-called negative capacitance (NC) behavior [17,32-34]. If a ferroelectric is incorporated as a gate dielectric within a field effect transistor (FET), this behavior should lead to enhancement of the surface potential at the semiconductor interface and reduction of the power consumption of the device, motivating tremendous interest in NC-FETs [35]. However, the presence of free carriers in the semiconductor modifies the electrostatic boundary conditions and may affect the domain structure in the ferroelectric. It is therefore important to understand nanoscale domain formation in ultrathin ferroelectrics in the presence of carriers. Here we report on the growth and structural characterization of ferroelectricmetal $\mathrm{PbTiO}_{3} / \mathrm{SrRuO}_{3}$ superlattices, and we investigate the appearance of nanoscale domains in the presence of metallic screening and their contribution to the dielectric response.

Unlike ferroelectric-dielectric superlattices, metalferroelectric superlattices are comparatively less well explored [36-40], with most of the attention focused on the properties of the metal rather than the ferroelectric [41-43]. Domain formation in $\mathrm{PbTiO}_{3} / \mathrm{SrRuO}_{3}$ superlattices has been reported by Callori et al. [44]; however, in this case the one-unit-cell-thick $\mathrm{SrRuO}_{3}$ layers were found to be insulating. On the other hand, ultrathin metal-ferroelectric-metal capacitors have been widely investigated, both theoretically and experimentally, and domain formation has frequently been invoked to explain the loss of stability of the 
monodomain state as the ferroelectric thickness is reduced. The appearance of domains is caused by the imperfect screening of the spontaneous polarization due to the finite intrinsic screening length at the metal-ferroelectric interface, and it is expected even for structurally perfect interfaces $[5,45,46]$. However, experimentally, the presence of domains was usually inferred indirectly from studies of polarization dynamics [47], film tetragonality [48], macroscopic piezoresponse [49,50], or second-harmonic generation [51]. Recently, depolarization-induced polarization arrangements [52] and periodic flux-closure structures [27] have been observed directly in electroded $\mathrm{PbTiO}_{3}$ films using transmission electron microscopy (TEM), but a systematic study of these domains, their scaling with ferroelectric thickness, and their effect on the macroscopic properties is still lacking.

In this work, we focus on the formation of $180^{\circ}$ ferroelectric domains in $\mathrm{PbTiO}_{3} / \mathrm{SrRuO}_{3}$ superlattices deposited on $\mathrm{SrTiO}_{3}$ substrates. Scanning TEM measurements show that the superlattices have two inequivalent interfaces resulting from the different growth modes of $\mathrm{PbTiO}_{3}$ and $\mathrm{SrRuO}_{3}$. We demonstrate that the ultrathin $\mathrm{SrRuO}_{3}$ layers remain metallic and that domains nevertheless appear within the $\mathrm{PbTiO}_{3}$ layers. Using $\mathrm{x}$-ray diffraction, we investigate the scaling of domain size with $\mathrm{PbTiO}_{3}$ layer thickness, and we find that the domain sizes are comparable to those in their counterpart ferroelectric-dielectric systems. Piezoresponse force microscopy measurements show that the domain structure in the $\mathrm{PbTiO}_{3}$ layers adopts a bubblelike configuration with sizes comparable to those determined from $\mathrm{x}$-ray diffraction measurements. Finally, electrical measurements of the dielectric response reveal a temperature dependence consistent with permittivity enhancement due to domain wall motion.

\section{EXPERIMENT}

$\mathrm{PbTiO}_{3} / \mathrm{SrRuO}_{3}$ superlattices were deposited on (001)oriented $\mathrm{SrTiO}_{3}$ substrates using off-axis radiofrequency magnetron sputtering. The $\mathrm{PbTiO}_{3}$ layers were grown in a 0.18 Torr atmosphere with an oxygen:argon ratio of 20:28; the corresponding parameters for $\mathrm{SrRuO}_{3}$ are 0.1 Torr and 3:60. During growth, the substrate was kept at a constant temperature of $550{ }^{\circ} \mathrm{C}$, as measured by a thermocouple inside the heating block. Although this temperature is suboptimal for the growth of $\mathrm{SrRuO}_{3}$, it was chosen to minimize $\mathrm{Pb}$ loss and optimize the electrical properties of the $\mathrm{PbTiO}_{3}$. In the following, the notation used to label the superlattices is $(\Lambda \text { u.c. })_{N}$, where $\Lambda$ is the superlattice wavelength/period in unit cells (u.c.) (the total number of unit cells in each repeating unit) and $N$ is the number of repetitions. The individual $\mathrm{PbTiO}_{3}$ and $\mathrm{SrRuO}_{3}$ thicknesses are then determined using a combination of x-ray diffraction and transmission electron microscopy, as will be discussed later in the paper.

Several series of $\mathrm{PbTiO}_{3} / \mathrm{SrRuO}_{3}$ superlattices were investigated, each with a fixed $\mathrm{SrRuO}_{3}$ layer thickness and varying thicknesses of $\mathrm{PbTiO}_{3}$. Structural characterization of the superlattices was conducted using a Rigaku Smartlab $\mathrm{x}$-ray diffractometer with a rotating anode. Atomic force microscopy (AFM) was used to characterize the surfaces of the superlattices, using a Bruker Dimension 3100 and Dimension
Icon microscopes. Piezoresponse force microscopy (PFM) measurements were performed using a Bruker Dimension Icon microscope. Low-temperature resistivity measurements were performed in a Janis CCS-150 closed-cycle helium cryostat between 8 and $300 \mathrm{~K}$. A $1 \mu \mathrm{A}$ current was supplied using a Keithley 2636B SourceMeter, and a Keithley 2000 Multimeter was used to measure the voltage in four-point geometry with the four corners of the sample contacted using silver paste. Sheet resistance values were calculated using the van der Pauw formula with the resistance values for the two orthogonal configurations obtained during separate cooling and heating runs. The reproducibility of the measurements was confirmed by repeating them multiple times.

Scanning transmission electron microscopy (STEM) measurements were performed on a lamella prepared by focused ion beam milling, using a FEI Helios 660 dual beam. The measurements were conducted using a FEI Titan ${ }^{3}$ G2 60-300 microscope, operating at $300 \mathrm{kV}$ in STEM mode.

For macroscopic capacitance measurements, circular pads with diameters of 50, 100, and $200 \mu \mathrm{m}$ were patterned using UV photolithography, and the samples were partially etched using Ar-ion milling. The remaining unetched $\mathrm{SrRuO}_{3}$ layers were used as a bottom contact accessed from the side of the sample using silver paste, whereas the top $\mathrm{SrRuO}_{3}$ layers were contacted using an indium wire pressed onto the surface of the circular $\mathrm{SrRuO}_{3}$ pads. Capacitance measurements as a function of frequency were conducted using an Agilent E4980A precision LCR meter over a frequency range from $100 \mathrm{~Hz}$ to $2 \mathrm{MHz}$, using an excitation voltage of $10 \mathrm{mV}$.

\section{RESULTS}

\section{A. Structural characterization}

In the absence of in situ growth monitoring, fabrication of superlattices with desired periodicities is challenging. When the deposition rates of the two components are constant throughout the growth process, desired layer thicknesses can be obtained by a careful pre-calibration of the growth rate for each material. However, initial characterization of the $\mathrm{PbTiO}_{3} / \mathrm{SrRuO}_{3}$ superlattices revealed that the growth rate of $\mathrm{SrRuO}_{3}$ is not constant, with the first few $\mathrm{SrRuO}_{3}$ monolayers growing significantly slower than the subsequent ones, making it challenging to obtain the desired $\mathrm{SrRuO}_{3}$ thickness based purely on growth time. We have therefore deposited four series of superlattices, each with fixed $\mathrm{SrRuO}_{3}$ deposition time per layer and varying deposition times for the $\mathrm{PbTiO}_{3}$ layers.

The results of x-ray diffraction measurements on samples from two such series are shown in Fig. 1. The presence of intense superlattice reflections and finite-size oscillations indicates that the samples have well-defined superlattice periodicities and total thickness. From the most intense superlattice peaks, the average pseudocubic lattice parameter can be determined, allowing the superlattice period $\Lambda$ to be expressed in terms of the total number of unit cells $\left(\mathrm{PbTiO}_{3}+\mathrm{SrRuO}_{3}\right)$ per period. This total number of unit cells per period is plotted as a function of $\mathrm{PbTiO}_{3}$ deposition time in Fig. 2.

The data for different $\mathrm{PbTiO}_{3}$ growth times $\left(t_{\mathrm{PTO}}\right)$ show a linear trend, indicating that for $t_{\mathrm{PTO}} \gtrsim 25 \mathrm{~min}$, the $\mathrm{PbTiO}_{3}$ 


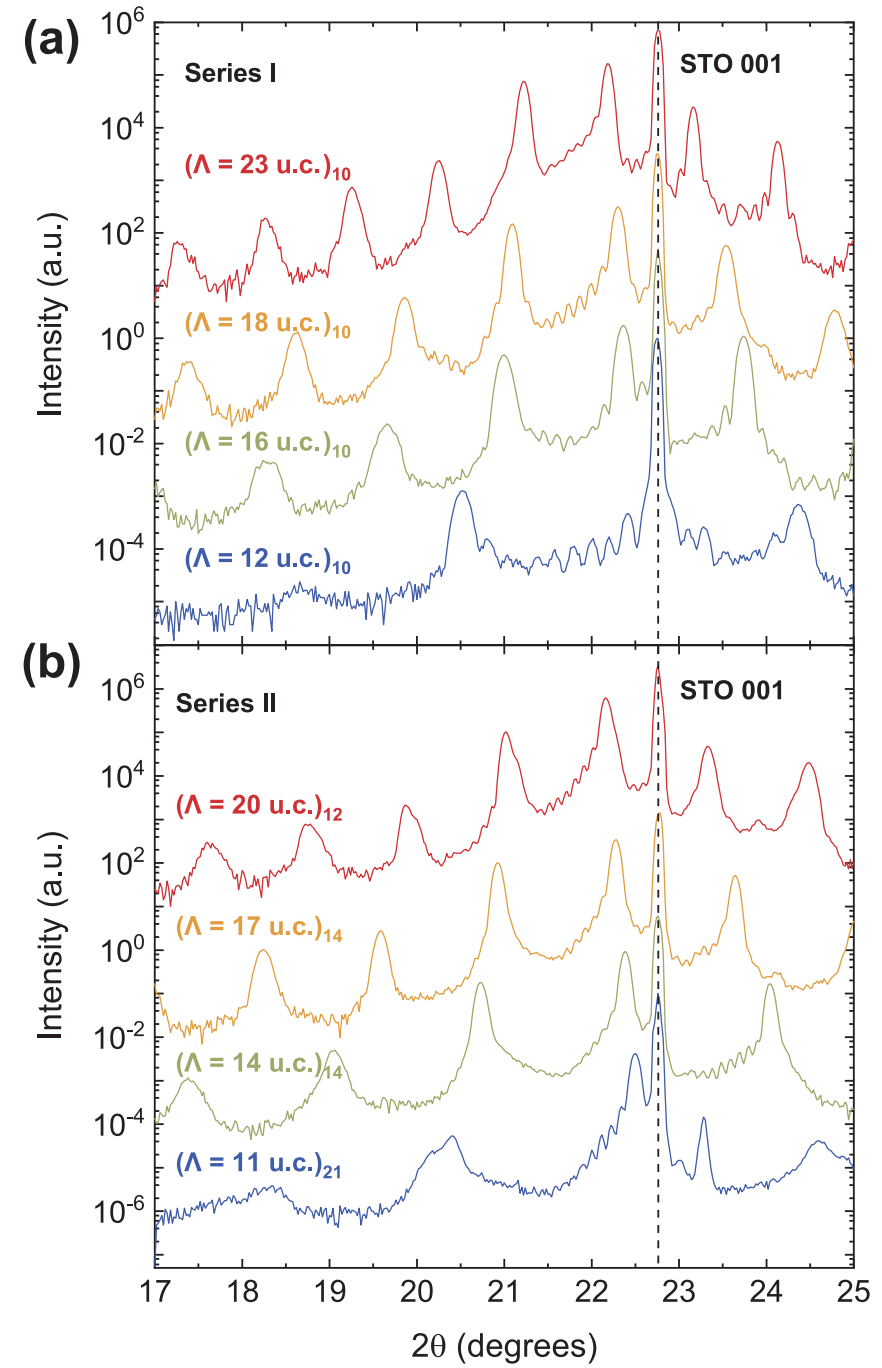

FIG. 1. $\theta-2 \theta$ scans of two series of $\mathrm{PbTiO}_{3} / \mathrm{SrRuO}_{3}$ superlattices around the (001) peak of $\mathrm{SrTiO}_{3}$. The superlattice series are fabricated by fixing the growth time of $\mathrm{SrRuO}_{3}$ and varying the thickness of the $\mathrm{PbTiO}_{3}$ layers. The notation used in the legend of the figure corresponds to $(\Lambda \text { u.c. })_{N}$, with $\Lambda$ the total number of unit cells in each superlattice period, and $N$ the number of superlattice repetitions.

growth rate is constant. A linear fit yields approximately the same slope $\left(\mathrm{PbTiO}_{3}\right.$ growth rate) for all four superlattice series. Since for each series the total $\mathrm{SrRuO}_{3}$ layer growth time $t_{\mathrm{SRO}}$ is the same, if the initial $\mathrm{PbTiO}_{3}$ growth rate in the interval $0<t_{\mathrm{PTO}}<25$ min were the same, then the $t=0$ intercept of the straight line fit would directly correspond to the $\mathrm{SrRuO}_{3}$ layer thickness. However, as we discuss later, this would give an erroneous result, as the initial growth rate for $\mathrm{PbTiO}_{3}$ is lower. This is illustrated by the dashed lines in Fig. 2, which show schematically one possible growth path for the initial period $0<t_{\text {PTO }}<25 \mathrm{~min}$, where no data points are available.

Despite the well-defined diffraction peaks and finite-size oscillations, the surface topography of the samples depends strongly on the thickness of the $\mathrm{PbTiO}_{3}$ layer, becoming smoother for thicker $\mathrm{PbTiO}_{3}$ layers. This is shown in Fig. 3 for different superlattices with the same $\mathrm{SrRuO}_{3}$ growth time.

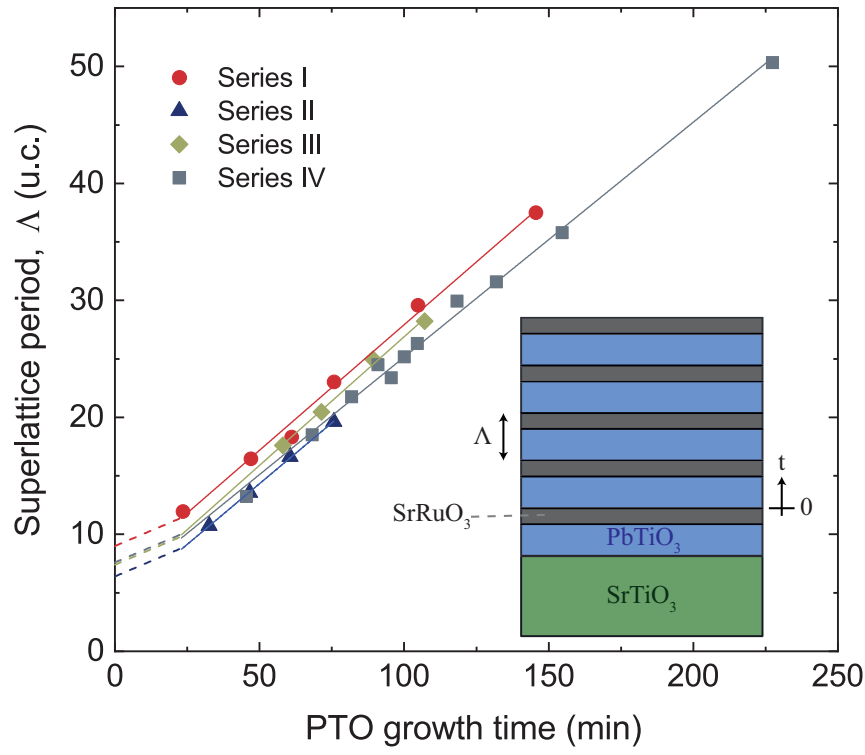

FIG. 2. Total number of unit cells in each superlattice period, $\Lambda$, plotted as a function of $\mathrm{PbTiO}_{3}$ layer growth time for all the series of $\mathrm{PbTiO}_{3} / \mathrm{SrRuO}_{3}$ superlattices presented in this study. Solid lines are linear fits, with slopes equal to $0.215,0.208,0.22$, and 0.201 $\mathrm{min} /$ u.c., respectively. The maximum error in the linear fits is 0.01 min/u.c. Dashed lines show one possible growth path for $\mathrm{PbTiO}_{3}$, for $t \lesssim 25 \mathrm{~min}$. The inset is a schematic of the superlattice structure, defining the characteristic period $\Lambda$.

The superlattice with a period of 11 u.c. exhibits a particulary rough surface with $\sim 20 \mathrm{~nm}$ high features. This roughness is also reflected in the less well-defined superlattice peaks for the corresponding sample in Fig. 1(b). By comparison, (a) $\quad(\Lambda=11 \text { u.c. })_{21}$

(c) $(\Lambda=17 \text { u.c. })_{14}$

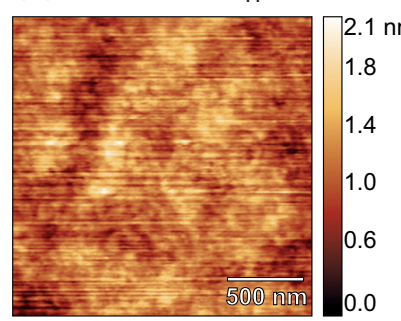

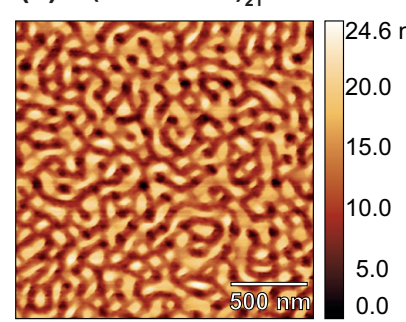

(b) $\quad(\Lambda=14 \text { u.c. })_{17}$

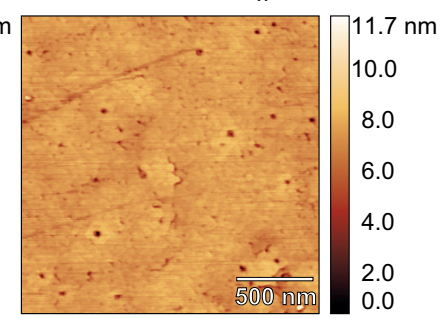

(d) $\quad(\Lambda=20 \text { u.c. })_{12}$

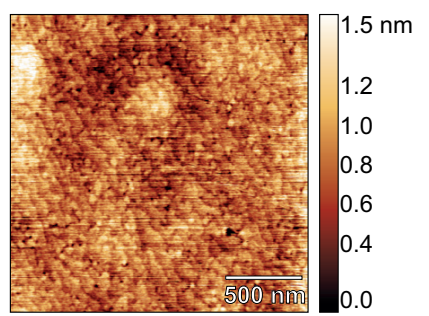

FIG. 3. Surface topography measured by AFM for $\mathrm{PbTiO}_{3} / \mathrm{SrRuO}_{3}$ superlattices in Series II, showing that the surface of the superlattices becomes smoother as the superlattice period (and therefore the $\mathrm{PbTiO}_{3}$ layer thickness) is increased. The average roughness values are (a) $3.70 \mathrm{~nm}$, (b) $0.38 \mathrm{~nm}$, (c) $0.32 \mathrm{~nm}$, and (d) $0.20 \mathrm{~nm}$. The height of the features in (a) is $\sim 20 \mathrm{~nm}$. 
(a)

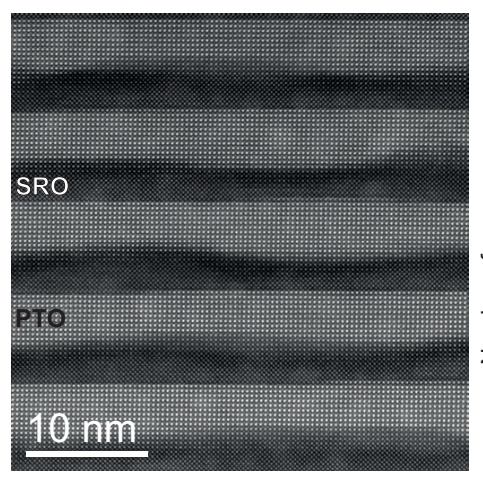

(b)

(c)

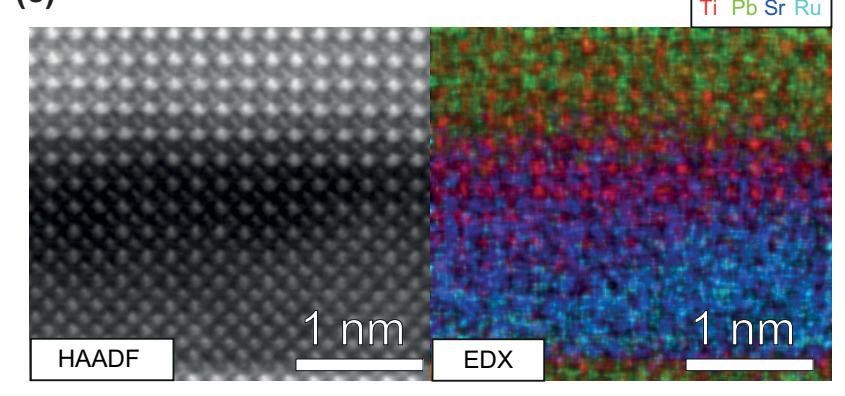

FIG. 4. STEM characterization of a $(\Lambda=20 \text { u.c. })_{12}$ $\mathrm{PbTiO}_{3} / \mathrm{SrRuO}_{3}$ superlattice. (a) HAADF image showing the asymmetry between two types of interface. (b) Histograms of the number of $\mathrm{PbTiO}_{3}$ unit cells in the sample measured at every (100) plane for each individual layer in image (a) (top) and for the whole sample across multiple images (bottom). The average $\mathrm{PbTiO}_{3}$ layer thickness is $11.0 \pm 1.5$ u.c. (c) High-magnification HAADF image and corresponding EDX map of a region that shows intermixing.

superlattices with periods above 14 u.c. display mean surface roughnesses below one unit cell, and sharper superlattice peaks [Fig. 1(b)].

The observed surface roughening is likely to be related to the growth mode of $\mathrm{SrRuO}_{3}$ on $\mathrm{PbTiO}_{3}$. Previous studies on $\mathrm{PbTiO}_{3} / \mathrm{SrRuO}_{3}$ superlattices deposited using off-axis radiofrequency magnetron sputtering show that a three-dimensional growth mode occurs for superlattices with $\mathrm{SrRuO}_{3}$ layers thicker than one unit cell [53]. This growth mode is most probably due to the lower temperatures used to grow the $\mathrm{SrRuO}_{3}$ layers $\left(550^{\circ} \mathrm{C}\right.$ instead of $\left.650{ }^{\circ} \mathrm{C}\right)$, which are required to preserve the quality of the $\mathrm{PbTiO}_{3}$ layers. The AFM scans show that there is a minimum $\mathrm{PbTiO}_{3}$ layer thickness required to make the superlattice surface flat, presumably corresponding to the amount of deposited $\mathrm{PbTiO}_{3}$ required to compensate (level out) the roughness of the $\mathrm{SrRuO}_{3}$ layers.

To investigate the interface structure, we have performed high-angle annular dark-field (HAADF) measurements on a $(\Lambda=20 \text { u.c. })_{12}$ superlattice from Series II, shown in Fig. 4(a). The HAADF image shows that the $\mathrm{PbTiO}_{3}$ interfaces are asymmetric [54], consistent with previous observations in $\mathrm{PbTiO}_{3} / \mathrm{SrRuO}_{3}$ superlattices with $\mathrm{SrRuO}_{3}$ thickness larger than one unit cell [53]. The top surface of each $\mathrm{PbTiO}_{3}$ layer is very flat, forming a sharp interface with
$\mathrm{SrRuO}_{3}$. By contrast, the bottom surface of each $\mathrm{PbTiO}_{3}$ layer is rough, due to the roughness of the underlying $\mathrm{SrRuO}_{3}$ layers. This roughness gives rise to a thickness variation of the $\mathrm{PbTiO}_{3}$ layers.

To quantify the thickness variation, we have determined the local thicknesses of $\mathrm{PbTiO}_{3}$ at every lateral position along each layer. To do so, we have counted the number of $\mathrm{Pb}$ columns within each (100) atomic plane using both HAADF images and energy dispersive x-ray spectroscopy (EDX) images. The statistical distributions of thicknesses for each individual layer in a single image, as well as across multiple HAADF images, are summarized by the histograms in Fig. 4. The extracted average $\mathrm{PbTiO}_{3}$ thickness for this sample is $11.0 \pm 1.5$ unit cells.

Further information can be obtained by examining the chemical compositions of the two types of interfaces using EDX. Figure 4(c) shows a high-magnification HAADF image (left) and an EDX intensity map (right) with different colors representing different atoms. While the sharp $\mathrm{SrRuO}_{3}-$ on- $\mathrm{PbTiO}_{3}$ interface is characterized by an abrupt change in cation composition from $(\mathrm{Sr}, \mathrm{Ru})$ to $(\mathrm{Pb}, \mathrm{Ti})$, the rougher $\mathrm{PbTiO}_{3}$-on-SrRuO 3 interface contains an intermediate region where $\mathrm{Sr}$ and $\mathrm{Ti}$ signals are present without significant $\mathrm{Ru}$ or $\mathrm{Pb}$ signals. The rougher interface therefore appears to contain intermediate $\mathrm{SrTiO}_{3}$-like regions approximately 2-3 u.c. thick, which can arise due to cation interdiffusion (intermixing).

The STEM measurements therefore show that each period of this superlattice consists of a continuous $\mathrm{SrRuO}_{3}$ layer that is approximately 4 u.c. thick, a complex broad interface, and a continuous $\mathrm{PbTiO}_{3}$ layer. The interface consists of rough islandlike $\mathrm{SrRuO}_{3}$ and $\mathrm{PbTiO}_{3}$ layers, as well as intermixed regions at their boundary. The $\mathrm{PbTiO}_{3}$ layer has a thickness that depends on its deposition time, which is $11.0 \pm 1.5$ u.c. in this case. Even though both x-ray diffraction and STEM give identical values for the measured period of the superlattice (20 u.c.), the average thickness of the $\mathrm{PbTiO}_{3}$ layers determined from STEM measurements is smaller than the thickness estimated assuming that $\mathrm{PbTiO}_{3}$ has a linear growth rate for all thicknesses. The initial growth rate of $\mathrm{PbTiO}_{3}$ for $t_{\text {PTO }}<25 \mathrm{~min}$ is therefore slower than that for $t_{\text {PTO }}>25 \mathrm{~min}$, as illustrated schematically by the dashed line in Fig. 2. We note that the initial growth phase of $\mathrm{PbTiO}_{3}$ is likely nonlinear, yet this cannot be concluded from the available data.

The variation in the $\mathrm{PbTiO}_{3}$ growth rate is probably related to the roughness of the $\mathrm{SrRuO}_{3}$ layer below and the interfacial intermixing. The STEM and AFM data suggest that the $\mathrm{PbTiO}_{3}$ growth rate becomes constant when the roughness of the $\mathrm{SrRuO}_{3}$ surface is leveled out by the first $\mathrm{PbTiO}_{3}$ layers. If the $\mathrm{PbTiO}_{3}$ thickness is not large enough, then cumulative roughness effects appear, as shown in Fig. 3(a).

The only difference between samples in the same series is the total $\mathrm{PbTiO}_{3}$ layer growth time $t_{\mathrm{PTO}}$, and for all samples

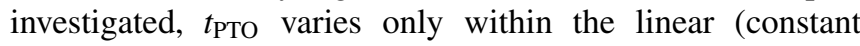
growth rate) regime. We can therefore use the $\mathrm{PbTiO}_{3}$ thickness obtained from STEM measurements for one of the samples and the calculated growth rate to determine the $\mathrm{PbTiO}_{3}$ thicknesses for all the other samples in the series. If we further assume that the initial growth phase of the $\mathrm{PbTiO}_{3}$ also follows the same behavior for samples with slightly 


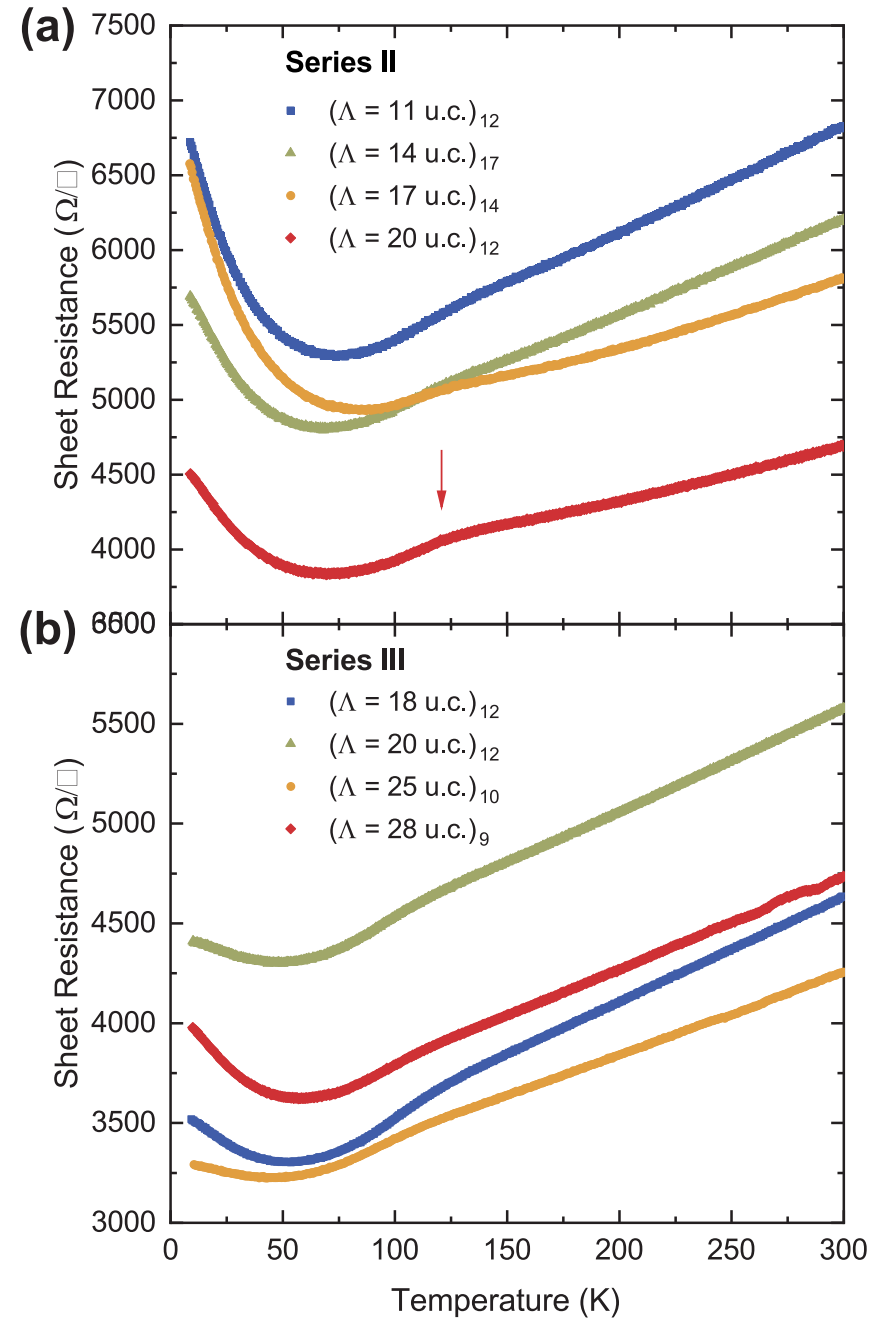

FIG. 5. Sheet resistance per superlattice period as a function of temperature for two series of $\mathrm{PbTiO}_{3} / \mathrm{SrRuO}_{3}$ superlattices. Each series in (a) and (b) has a fixed $\mathrm{SrRuO}_{3}$ growth time and varying $\mathrm{PbTiO}_{3}$ thickness. The red arrow marks the approximate ferromagnetic transition temperature in $\mathrm{SrRuO}_{3}$.

thicker or thinner $\mathrm{SrRuO}_{3}$, then the $\mathrm{PbTiO}_{3}$ thicknesses for samples in other series can also be determined.

\section{B. Electrical properties of $\mathrm{SrRuO}_{3}$ layers}

It is well known that ultrathin metallic oxides can undergo a metal-to-insulator transition below a certain critical thickness [55]. It is therefore imperative to check whether the thin $\mathrm{SrRuO}_{3}$ layers in our superlattices remain metallic.

Figure 5 shows the in-plane sheet resistance per superlattice period for two series of superlattices with a fixed thickness of $\mathrm{SrRuO}_{3}$ and varying $\mathrm{PbTiO}_{3}$ thickness. For all superlattices, the resistance of the $\mathrm{SrRuO}_{3}$ layers is observed to decrease with decreasing temperature, confirming metallic behavior down to temperatures of 50-100 K. In most samples, a change in gradient of the resistance-temperature curve associated with the ferromagnetic transition in $\mathrm{SrRuO}_{3}$ can also be discerned around $120 \mathrm{~K}$ [marked by the red arrow in Fig. 5(a)] [56,57]. The calculated room-temperature resistivity values for the $\mathrm{SrRuO}_{3}$ layers lie within a range

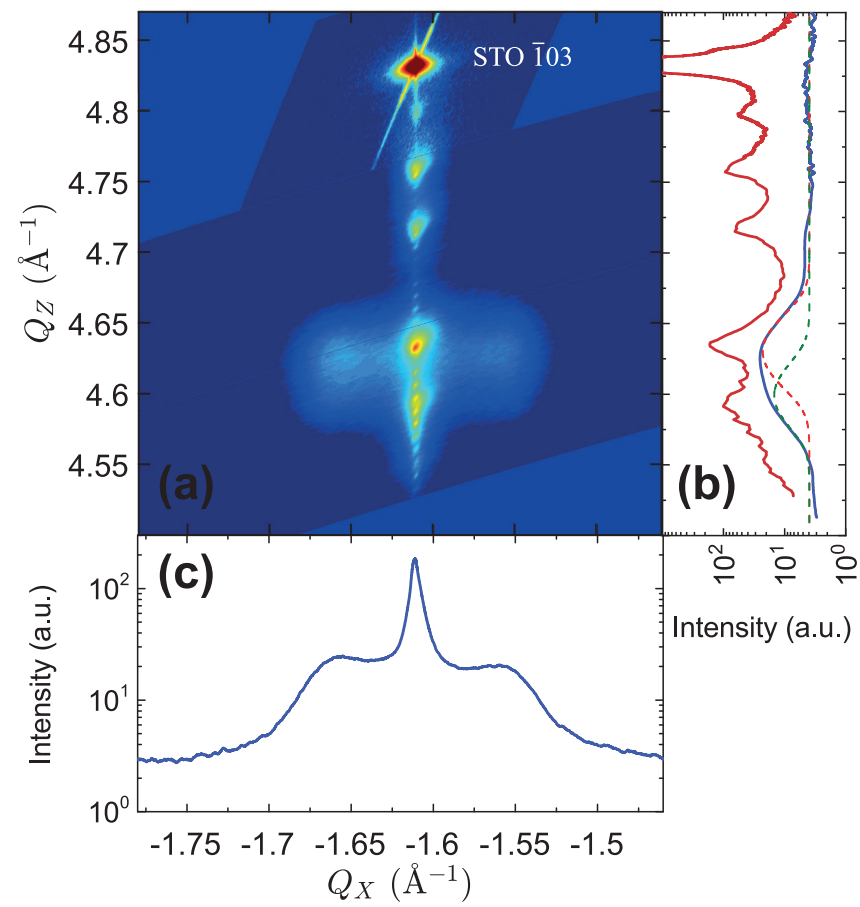

FIG. 6. (a) Reciprocal space map around the (103) peak of $\mathrm{SrTiO}_{3}$ for a $(\Lambda=37 \text { u.c. })_{8}$ superlattice with estimated 26 u.c. of $\mathrm{PbTiO}_{3}$ per repetition, showing domain satellites around the main superlattice Bragg peak. (b) Intensity profiles along $Q_{Z}$ at the $Q_{X}$ position of the main superlattice Bragg peaks (red) and the domain peaks (blue). The dashed curves are Gaussian fits to the domain peak profile. (c) Intensity profile along $Q_{X}$ across a superlattice Bragg peak and two domain satellite peaks.

of 700-2200 $\mu \Omega \mathrm{cm}$ (this wide range reflects primarily the large uncertainty in the $\mathrm{SrRuO}_{3}$ layer thickness due to the roughness, rather than intersample variation). These resistivity values are higher than those typical of thick $\mathrm{SrRuO}_{3}$ films, but they are similar to the values reported for highquality films of similar thickness [55]. At temperatures below 50-100 K we observe an upturn in the resistivity, consistent with previous reports on ultrathin $\mathrm{SrRuO}_{3}$ [58].

Assuming a $\mathrm{PbTiO}_{3}$ polarization of order $75 \mu \mathrm{C} / \mathrm{cm}^{2}$ for a film strained on $\mathrm{SrTiO}_{3}$ [59], full screening requires a charge density of $4.7 \times 10^{14} \mathrm{~cm}^{-2}$. The typical carrier density in $\mathrm{SrRuO}_{3}$ is around $4 \times 10^{22} \mathrm{~cm}^{-3}$ [60], giving a carrier density of approximately $16 \times 10^{14} \mathrm{~cm}^{-2}$ per monolayer of $\mathrm{SrRuO}_{3}$, more than three times the value required to screen the polarization. Therefore, even a single $\mathrm{SrRuO}_{3}$ monolayer should be sufficient to screen the polarization of $\mathrm{PbTiO}_{3}$.

\section{Domain structure in the $\mathrm{PbTiO}_{3}$ layers}

Figure 6(a) shows a reciprocal space map of a $(\Lambda=$ 37 u.c. $)_{8}$ superlattice with an estimated 26 u.c. of $\mathrm{PbTiO}_{3}$ around the (103) peak of $\mathrm{SrTiO}_{3}$. Clear signatures of ferroelectric domain formation are evident, manifested as a set of broad in-plane satellites around the main superlattice Bragg peak. This is consistent with a $180^{\circ}$ domain structure, as expected for compressively strained $\mathrm{PbTiO}_{3}$ on $\mathrm{SrTiO}_{3}$, and in agreement with previous observations in $\mathrm{PbTiO}_{3}$ thin 


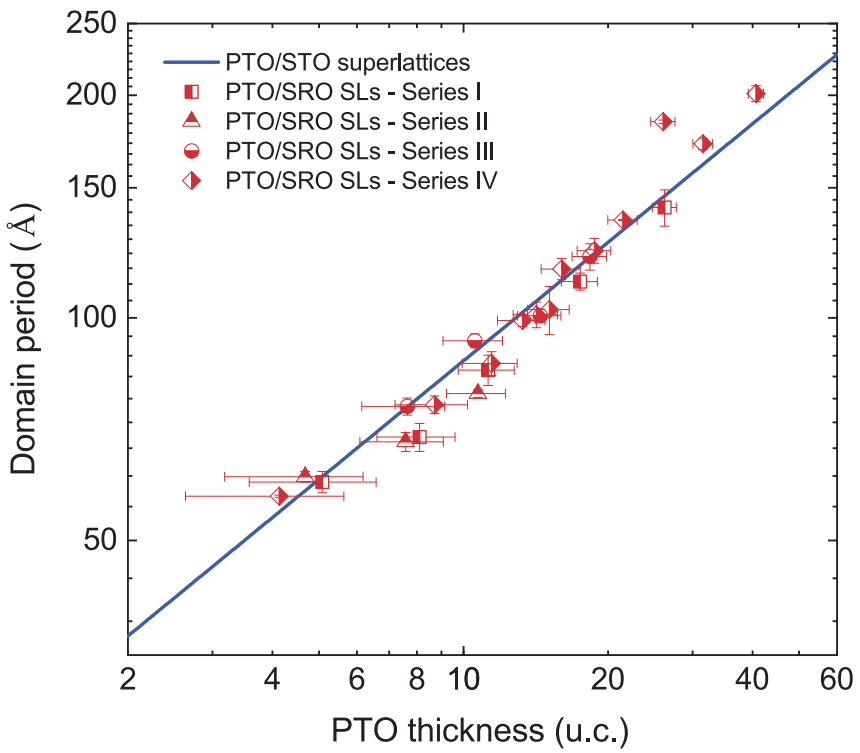

FIG. 7. Domain periods for $\mathrm{PbTiO}_{3} / \mathrm{SrRuO}_{3}$ superlattices as a function of estimated $\mathrm{PbTiO}_{3}$ layer thickness with both axes on the logarithmic scale. Red squares, triangles, circles, and diamonds show the domain periods for different series of superlattices, as described in the main text. Vertical error bars are determined by the uncertainty in the position of the Gaussian used to fit the domain satellites (see the main text). Horizontal error bars for all samples are assumed to be the same as the $(\Lambda=20 \text { u.c. })_{12}$ superlattice characterized using STEM ( \pm 1.5 u.c.). The solid line shows the scaling observed for $\mathrm{PbTiO}_{3} / \mathrm{SrTiO}_{3}$ superlattices fabricated using the same sample deposition system and $\mathrm{PbTiO}_{3}$ growth conditions.

films [61] and $\mathrm{PbTiO}_{3}$-based multilayers [11,12]. The observation of these domains implies that despite the presence of metallic layers with sufficient density of carriers, the ferroelectric polarization is not fully screened.

The intensity profiles of the domain satellite peaks along $Q_{Z}$ and $Q_{X}$ [Figs. 6(b) and 6(c), respectively] show that the satellites are broad in both directions in reciprocal space, indicating a small out-of-plane and in-plane coherence length. The domain satellites are fitted by Gaussian functions to determine their peak positions, widths, and the corresponding fitting uncertainties. From the positions of the domain satellites, the periodicity of the domain structure is found to be approximately $14 \mathrm{~nm}$. Using the Scherrer equation, the out-ofplane coherence length $\xi_{z} \approx 10 \mathrm{~nm}$ is obtained from the full width at half-maximum (FWHM), and it is comparable to the $\mathrm{PbTiO}_{3}$ layer thickness within each repetition $(11 \mathrm{~nm})$, indicating that the domain structures of adjacent $\mathrm{PbTiO}_{3}$ layers are decoupled.

Figure 7 illustrates how the domain periodicity depends on the $\mathrm{PbTiO}_{3}$ layer thickness for multiple samples, and compares it with the corresponding Landau-Lifshitz-Kittel scaling observed in $\mathrm{PbTiO}_{3} / \mathrm{SrTiO}_{3}$ superlattices fabricated with the same thin film deposition system, using the same $\mathrm{PbTiO}_{3}$ ceramic targets and growth parameters (scaling exponent equal to 0.5$)[62,63]$. We note that the vertical error bars in the domain periods in Fig. 7 correspond to uncertainties obtained from fitting the satellite peak positions, and they do not accurately capture the uncertainties due to local variations in domain sizes [64]. We find that the domains in our systems have similar sizes and scaling with thickness as in ferroelectric-dielectric $\mathrm{PbTiO}_{3} / \mathrm{SrTiO}_{3}$ superlattices, implying a comparable degree of screening in both systems. Thus, despite the metallic nature of the $\mathrm{SrRuO}_{3}$ layers, the screening of the polarization remains incomplete.

One reason for this incomplete screening may be the rough $\mathrm{PbTiO}_{3}-\mathrm{SrRuO}_{3}$ interface, where the intermixed $\mathrm{SrTiO}_{3}$-like layer can induce a large depolarizing field [22], while the roughness of the interface itself can lead to inhomogeneous screening. However, if the $\mathrm{SrTiO}_{3}$-like layer were the main contribution and the abrupt $\mathrm{PbTiO}_{3}-\mathrm{SrRuO}_{3}$ interface were perfectly screened, we would expect our domain sizes to be significantly larger than those found in $\mathrm{PbTiO}_{3} / \mathrm{SrTiO}_{3}$ superlattices with two such $\mathrm{PbTiO}_{3}-\mathrm{SrTiO}_{3}$ interfaces $[65,66]$. Instead, the observed domain sizes are similar to those in $\mathrm{PbTiO}_{3} / \mathrm{SrTiO}_{3}$ superlattices, implying that screening from the sharp $\mathrm{PbTiO}_{3}-\mathrm{SrRuO}_{3}$ interface is not sufficient either. This is consistent with previous observations in ultrathin $\mathrm{SrRuO}_{3} / \mathrm{BaTiO}_{3} / \mathrm{SrRuO}_{3}$ capacitors [50], as well as first-principles calculations [5,45], which predict that even structurally perfect $\mathrm{PbTiO}_{3}-\mathrm{SrRuO}_{3}$ interfaces have a finite intrinsic screening length that promotes domain formation.

\section{Piezoresponse force microscopy}

To image the domain structure directly, we have fabricated a $(\Lambda=37 \text { u.c. })_{8}$ superlattice with an estimated 26 u.c. of $\mathrm{PbTiO}_{3}$ in which the starting layer is $\mathrm{SrRuO}_{3}$ and the topmost layer is $\mathrm{PbTiO}_{3}$. Although the electrostatic boundary conditions for this topmost $\mathrm{PbTiO}_{3}$ layer with an exposed surface are not the same as for the inner layers that are sandwiched between two $\mathrm{SrRuO}_{3}$ layers, we can nevertheless extract useful information about domain formation in the superlattices.

The results of the PFM characterization are summarized in Fig. 8. The PFM amplitude and phase images [Figs. 8(a) and 8(b), respectively] reveal bubblelike domains, delineated by clear minima in the amplitude, and $180^{\circ}$ change of phase across the domain walls. Similar bubble domains were previously reported in $\mathrm{PbTiO}_{3} / \mathrm{SrTiO}_{3}$ and $\mathrm{PbZr}_{0.2} \mathrm{Ti}_{0.8} \mathrm{O}_{3} / \mathrm{SrTiO}_{3}$ heterostructures on $\mathrm{SrTiO}_{3}$ substrates [22,28]. We note that the topography of the sample, shown in Fig. 8(c), has a roughness of less than one unit cell.

Figure 8(d) shows the radially averaged autocorrelation function of the PFM amplitude image. The first minimum and the first maximum of the autocorrelation function can be used to determine the average feature size and the spacing between features in Fig. 8(b), respectively [67]. The average size of the individual bubbles can also be directly measured from the PFM amplitude image, and it is found to be approximately $13 \mathrm{~nm}$. The domain period of this superlattice, determined using XRD, is $16 \mathrm{~nm}$. These two sizes are marked with a red dashed line and a green dotted line, respectively, in Fig. 8(d), showing good agreement with the average feature size and spacing determined from the autocorrelation function. 
(a) Amplitude

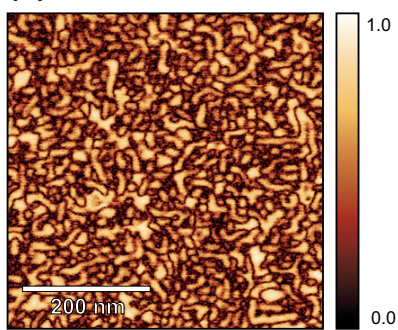

(c) Topography

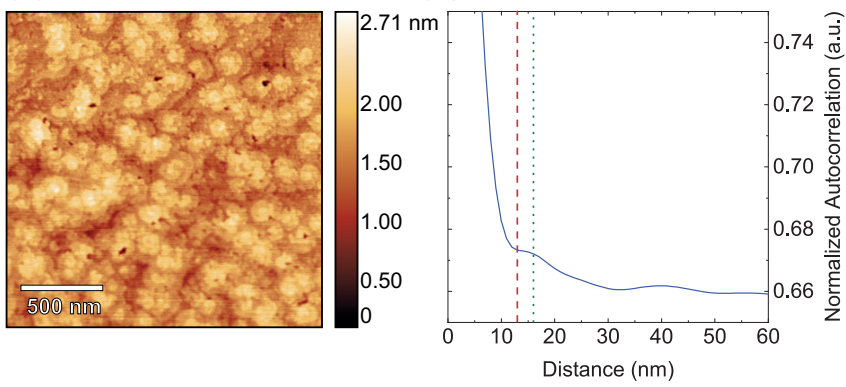

FIG. 8. Piezoresponse force microscopy (a) amplitude and (b) phase of a $(\Lambda=37 \text { u.c. })_{8}$ superlattice with an estimated 26 unit cells of $\mathrm{PbTiO}_{3}$ per period, in which the last grown layer is a $\mathrm{PbTiO}_{3}$ layer. The scans show a $180^{\circ}$ ferroelectric bubblelike domain structure with average bubble size equal to $13 \mathrm{~nm}$. (c) Largerarea topography scan, with average roughness equal to $0.22 \mathrm{~nm}$. (d) Normalized radially averaged autocorrelation function of the PFM amplitude image in (b), with the red dashed line showing the average bubble size $(13 \mathrm{~nm})$ and the green dotted line showing the domain period measured using XRD $(16 \mathrm{~nm})$. The two lines coincide with the first minimum and maximum in the autocorrelation function, respectively.

\section{E. Dielectric properties of $\mathrm{PbTiO}_{3}$ layers}

The conducting $\mathrm{SrRuO}_{3}$ layers allow the capacitance of the $\mathrm{PbTiO}_{3}$ layers in the superlattices to be measured. The frequency-dependent complex capacitance $C^{*}(\omega)$, defined in terms of the measured complex impedance $Z(\omega)$ as $C^{*}(\omega)=$ $C^{\prime}(\omega)+i C^{\prime \prime}(\omega)=1 / i \omega Z(\omega)$, is shown in Fig. 9 for a $(\Lambda=$ 30 u.c. $)_{10}$ superlattice with estimated 17 u.c. of $\mathrm{PbTiO}_{3}$ at different temperatures. The real part $C^{\prime}(\omega)$ exhibits a series of plateaus and relaxations, which are accompanied by the corresponding maxima in the dielectric loss $C^{\prime \prime}(\omega)$. This Maxwell-Wagner-type behavior is characteristic of a series arrangement of leaky capacitors, and it has previously been reported in ferroelectric-dielectric [68] and ferroelectricmetal [37] superlattices.

As the capacitance $C$ of each ferroelectric layer is expected to be the same, the different relaxations arise from differences in their resistances $R_{i}$, implying that leakage through each $\mathrm{PbTiO}_{3}$ layer is likely to be due to localized conducting pathways rather than homogeneous conduction across the entire layer. To extract the individual layer capacitances and resistances, the data are fitted to an equivalent circuit model consisting of parallel $R C$ elements connected in series, with an additional series resistance accounting for the contribution of the contacts as shown in the inset of Fig. 9(b). The number of series $R C$ elements is determined independently using two

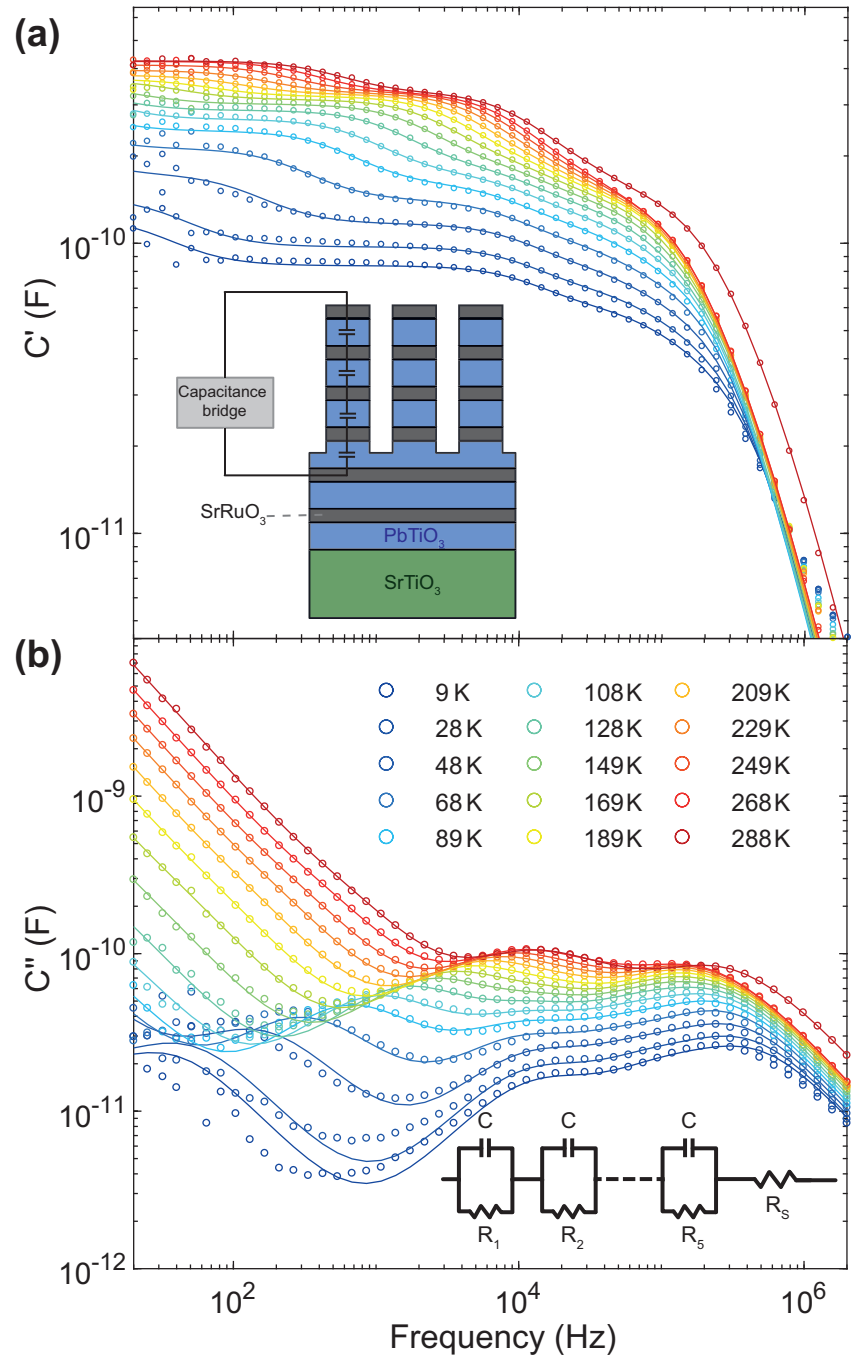

FIG. 9. Frequency dependence of the real (a) and imaginary (b) parts of the complex capacitance for a $(\Lambda=30 \text { u.c. })_{10}$ superlattice with estimated 17 unit cells of $\mathrm{PbTiO}_{3}$ per repetition. Open circles correspond to the data, whereas solid lines represent the fits to the equivalent circuit shown in (b), which consists of five parallel $\mathrm{RC}$ elements connected in series and an additional series resistance due to contacts and connections. A schematic of the measurement setup is shown as an inset in (a).

methods. First, the depth of the etched regions of the superlattice is measured using AFM, allowing the number of etched superlattice periods to be determined. The number of etched periods is then cross-checked by fitting the data for a large number of capacitor stacks on the same sample to models with more and fewer $R C$ elements. Only the model with the correct number of elements gives a consistent value of $C$ (within approx. $10 \%$ variation) for all the capacitor stacks. For the case of Fig. 9, five $R C$ elements are required.

In general, the fits reproduce the experimental data well. Nevertheless, some features of the data are not captured. First, any information on the dispersion of the real part of the capacitance is lost. Most real dielectric systems exhibit a frequency-dependent permittivity, which is associated with the dynamics of the excited physical process [69]. By assuming that all our circuit elements are ideal capacitors, this 


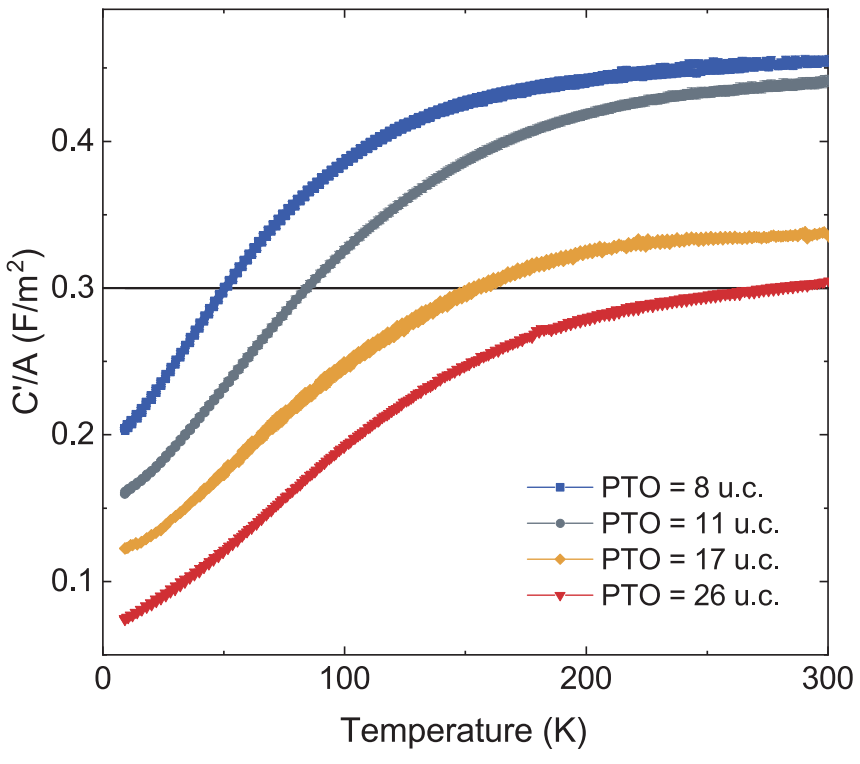

FIG. 10. Fitted values of the capacitance per unit area for $\mathrm{PbTiO}_{3}$ layers with estimated thickness equal to $8,11,17$, and 26 u.c. as a function of temperature. The horizontal line corresponds to the theoretically predicted interface contribution (from density functional theory calculations at $0 \mathrm{~K} \mathrm{[46]).}$

frequency dependence is neglected. Using dispersive elements (such as the constant phase element [70]) improves the fit; however, it leads to an overparametrization of the model, and the extracted fitting parameters become less reliable. Second, any intrinsic dielectric loss information, which is associated with the physical processes in our samples (e.g., freezing of the domain walls [71]), is also lost because the loss is dominated by the conductivity of the $\mathrm{PbTiO}_{3}$ layers. Finally, we note that any inductive effect in the response of the system due to the length of the measurement cables or the lowtemperature ferromagnetic phase of $\mathrm{SrRuO}_{3}$ is negligible and is not required to fit the observed frequency response.

Using the fitting procedure above, the capacitance of individual $\mathrm{PbTiO}_{3}$ layers is obtained as a function of temperature for superlattices with different $\mathrm{PbTiO}_{3}$ layer thicknesses and is shown in Fig. 10. In the case of the superlattice with 26 u.c. of $\mathrm{PbTiO}_{3}$, the measured capacitor was highly insulating so there was no need to fit the impedance spectra, and the $1 \mathrm{kHz}$ values were used. For superlattices with 11 u.c. of $\mathrm{PbTiO}_{3}$, identical behavior of the capacitance was obtained for two different samples from different series, confirming its reproducibility and the lack of dependence on the $\mathrm{SrRuO}_{3}$ layer thickness (the extracted capacitances for the two samples were within the errors associated with the fitting process, and within $2 \%$ of each other). The error bars from the fits are also plotted but are very small compared to the scatter and the size of the markers. We note that, whenever possible, multiple capacitors were measured for each sample to confirm the reproducibility of the observed behavior.

For all samples, the capacitance is weakly temperaturedependent near room temperature but decreases sharply at low temperatures. The concave shape of the curves is distinct from the convex behavior expected from the intrinsic lattice (phonon) response, but it is qualitatively similar to the behavior observed in a range of polydomain systems in- cluding bulk potassium dihydrogen phosphate and triglycine sulfate [71], $\mathrm{CaTiO}_{3} / \mathrm{SrTiO}_{3} / \mathrm{BaTiO}_{3}$ superlattices [72], and ultrathin $\mathrm{BaTiO}_{3}$ films [50]. With the presence of domains confirmed by $\mathrm{x}$-ray diffraction, we attribute this response to domain dynamics, which relax out at low temperature due to domain wall pinning. It appears that the temperature at which this relaxation occurs is lower for smaller $\mathrm{PbTiO}_{3}$ thicknesses, but due to the broad nature of the relaxation and the absence of useful loss information, it is difficult to quantify this thickness dependence and extract the thermal activation energies characterizing this process.

Due to the large uncertainty in the individual $\mathrm{PbTiO}_{3}$ layer thicknesses and the presence of the intermixed layer, which contributes a series capacitance, we refrain from calculating the dielectric constant of each layer. Nevertheless, it is still instructive to compare the measured capacitance to the expected value of the interface capacitance for a $\mathrm{PbTiO}_{3}-\mathrm{SrRuO}_{3}$ interface. First-principles calculations predict the latter to be $\sim 0.6 \mathrm{~F} / \mathrm{m}^{2}$ [46], and the capacitance of two such interface layers in series with each $\mathrm{PbTiO}_{3}$ layer is shown by the horizontal line in Fig. 10. Interestingly, there exists a temperature regime for which the total measured capacitance is higher than that of the overall interface capacitance, signifying that the effective capacitance of the $\mathrm{PbTiO}_{3}$ layer is negative. This seems counterintuitive, yet it is consistent with previous theoretical and experimental work, in which the local effective permittivity of the ferroelectric becomes negative due to domain wall motion $[17,32,50]$. We note that this qualitative conclusion is unaffected by the presence of the intermixed layer, as (i) the theoretically predicted $\mathrm{SrRuO}_{3}-\mathrm{SrTiO}_{3}$ interface capacitance is similar to that of $\mathrm{SrRuO}_{3}-\mathrm{PbTiO}_{3}$ [46], and (ii) any intermixed layer contributes as another capacitance in series, which only reduces (rather than increases) the overall interface capacitance and therefore broadens the temperature range of the negative permittivity region deduced from the data.

However, the above conclusion is strongly dependent on the assumed value for the interface capacitance. The observed capacitance values could be explained without invoking ferroelectric negative capacitance if the interface capacitance is at least a factor of 1.5 larger than the theoretically predicted value. Interface capacitances around $1 \mathrm{~F} / \mathrm{m}^{2}$ have been previously extracted for $\mathrm{Ba}_{0.7} \mathrm{Sr}_{0.3} \mathrm{TiO}_{3} / \mathrm{SrRuO}_{3}$ [73] and $\mathrm{SrTiO}_{3} / \mathrm{SrRuO}_{3}$ [17] interfaces. If the $\mathrm{PbTiO}_{3} / \mathrm{SrRuO}_{3}$ interface has a similar capacitance, then the capacitance of the $\mathrm{PbTiO}_{3}$ layers would be positive. Nevertheless, even in this case, the corresponding effective permittivity of the $\mathrm{PbTiO}_{3}$ layers (of the order 1000-2000, depending on the $\mathrm{PbTiO}_{3}$ layer thickness) is still strongly enhanced due to domain wall motion. For a more accurate estimate of the local $\mathrm{PbTiO}_{3}$ permittivity, a more detailed investigation of the interface capacitance is needed.

\section{CONCLUSIONS}

In summary, we have fabricated ferroelectric-metal $\mathrm{PbTiO}_{3} / \mathrm{SrRuO}_{3}$ superlattices with varying $\mathrm{PbTiO}_{3}$ and $\mathrm{SrRuO}_{3}$ thicknesses on single-crystalline $\mathrm{SrTiO}_{3}$ substrates. Scanning transmission electron microscopy measurements have shown that the interfaces between the two materials are asymmetric due to the different growth modes of $\mathrm{PbTiO}_{3}$ 
and $\mathrm{SrRuO}_{3}$. Using x-ray diffraction, ferroelectric domains were observed with sizes comparable to those found in $\mathrm{PbTiO}_{3} / \mathrm{SrTiO}_{3}$ superlattices. Therefore, despite the metallic nature of the ultrathin $\mathrm{SrRuO}_{3}$ layers, domains appear due to the depolarizing field caused by the imperfect screening of the polarization at the $\mathrm{PbTiO}_{3} / \mathrm{SrRuO}_{3}$ interfaces. Piezoresponse force microscopy measurements show that the domain structure in the topmost $\mathrm{PbTiO}_{3}$ layers adopts a bubblelike configuration with sizes comparable to those determined from $\mathrm{X}$-ray diffraction measurements. The dielectric response of individual $\mathrm{PbTiO}_{3}-\mathrm{SrRuO}_{3}$ capacitors within the superlattice stacks was measured for ultrathin $\mathrm{PbTiO}_{3}$ layers, revealing a temperature-dependent capacitance enhancement expected from the contribution of domain wall motion.
The datasets used in this publication are available from the corresponding author on reasonable request.

\section{ACKNOWLEDGMENTS}

We gratefully acknowledge funding from the EPSRC (Grants No. EP/M007073/1 and No. EP/S010769/1, and studentship No. 1447654), the China Scholarship Council, and the A.G. Leventis Foundation. STEM work was carried out within the MATMECA consortium and supported by the ANR under Contract No. ANR-10-EQPX-37. It has benefited from the facilities of the Laboratory MSSMat, CNRS, CentraleSupélec, Université Paris-Saclay, France.
[1] E. D. Specht, H.-M. Christen, D. P. Norton, and L. A. Boatner, Phys. Rev. Lett. 80, 4317 (1998).

[2] D. D. Fong, G. B. Stephenson, S. K. Streiffer, J. A. Eastman, O. Auciello, P. H. Fuoss, and C. Thompson, Science 304, 1650 (2004).

[3] P. Zubko, N. Stucki, C. Lichtensteiger, and J.-M. Triscone, Phys. Rev. Lett. 104, 187601 (2010).

[4] V. A. Stephanovich, I. A. Luk'yanchuk, and M. G. Karkut, Phys. Rev. Lett. 94, 047601 (2005).

[5] P. Aguado-Puente and J. Junquera, Phys. Rev. Lett. 100, 177601 (2008).

[6] S. Lisenkov, I. Ponomareva, and L. Bellaiche, Phys. Rev. B 79, 024101 (2009).

[7] Q. Zhang, R. Herchig, and I. Ponomareva, Phys. Rev. Lett. 107, 177601 (2011).

[8] Z. Jiang, R. Zhang, D. Wang, D. Sichuga, C.-L. Jia, and L. Bellaiche, Phys. Rev. B 89, 214113 (2014).

[9] J. B. J. Chapman, A. V. Kimmel, and D. M. Duffy, Phys. Chem. Chem. Phys. 19, 4243 (2017).

[10] J. Y. Jo, P. Chen, R. J. Sichel, S. J. Callori, J. Sinsheimer, E. M. Dufresne, M. Dawber, and P. G. Evans, Phys. Rev. Lett. 107, 055501 (2011).

[11] P. Zubko, N. Jecklin, N. Stucki, C. Lichtensteiger, G. Rispens, and J.-M. Triscone, Ferroelectrics 433, 127 (2012).

[12] P. Zubko, N. Jecklin, A. Torres-Pardo, P. Aguado-Puente, A. Gloter, C. Lichtensteiger, J. Junquera, O. Stéphan, and J.-M. Triscone, Nano Lett. 12, 2846 (2012).

[13] J. Sinsheimer, S. J. Callori, B. Bein, Y. Benkara, J. Daley, J. Coraor, D. Su, P. W. Stephens, and M. Dawber, Phys. Rev. Lett. 109, 167601 (2012).

[14] N. Lemée, I. C. Infante, C. Hubault, A. Boulle, N. Blanc, N. Boudet, V. Demange, and M. G. Karkut, ACS Appl. Mater. Interfaces 7, 19906 (2015).

[15] B. Bein, H.-C. Hsing, S. J. Callori, J. Sinsheimer, P. V. Chinta, R. L. Headrick, and M. Dawber, Nat. Commun. 6, 10136 (2015).

[16] Y. Ahn, J. Park, A. Pateras, M. B. Rich, Q. Zhang, P. Chen, M. H. Yusuf, H. Wen, M. Dawber, and P. G. Evans, Phys. Rev. Lett. 119, 057601 (2017).

[17] P. Zubko, J. C. Wojdeł, M. Hadjimichael, S. Fernandez-Pena, A. Sené, I. Luk'yanchuk, J.-M. Triscone, and J. Íñiguez, Nature (London) 534, 524 (2016).

[18] A. Boulle, I. C. Infante, and N. Lemée, J. Appl. Crystallogr. 49, 845 (2016).
[19] M. Hadjimichael, E. Zatterin, S. Fernandez-Peña, S. J. Leake, and P. Zubko, Phys. Rev. Lett. 120, 037602 (2018).

[20] J. Park, J. Mangeri, Q. Zhang, M. H. Yusuf, A. Pateras, M. Dawber, M. V. Holt, O. G. Heinonen, S. Nakhmanson, and P. G. Evans, Nanoscale 10, 3262 (2018).

[21] V. A. Stoica, N. Laanait, C. Dai, Z. Hong, Y. Yuan, Z. Zhang, S. Lei, M. R. McCarter, A. Yadav, A. R. Damodaran, S. Das, G. A. Stone, J. Karapetrova, D. A. Walko, X. Zhang, L. W. Martin, R. Ramesh, L.-Q. Chen, H. Wen, V. Gopalan, and J. W. Freeland, Nat. Mater. 18, 377 (2019).

[22] C. Lichtensteiger, S. Fernandez-Pena, C. Weymann, P. Zubko, and J.-M. Triscone, Nano Lett. 14, 4205 (2014).

[23] C. Lichtensteiger, C. Weymann, S. Fernandez-Pena, P. Paruch, and J.-M. Triscone, New J. Phys. 18, 043030 (2016).

[24] A. R. Damodaran, J. D. Clarkson, Z. Hong, H. Liu, A. K. Yadav, C. T. Nelson, S. L. Hsu, M. R. McCarter, K. D. Park, V. Kravtsov, A. Farhan, Y. Dong, Z. Cai, H. Zhou, P. AguadoPuente, P. Garcia-Fernandez, J. Iniguez, J. Junquera, A. Scholl, M. B. Raschke, L. Q. Chen, D. D. Fong, R. Ramesh, and L. W. Martin, Nat. Mater. 16, 1003 (2017).

[25] Y. L. Tang, Y. L. Zhu, X. L. Ma, A. Y. Borisevich, A. N. Morozovska, E. A. Eliseev, W. Y. Wang, Y. J. Wang, Y. B. Xu, Z. D. Zhang, and S. J. Pennycook, Science 348, 547 (2015).

[26] Y. Liu, Y.-J. Wang, Y.-L. Zhu, C.-H. Lei, Y.-L. Tang, S. Li, S.-R Zhang, J. Li, and X.-L. Ma, Nano Lett. 17, 7258 (2017).

[27] S. Li, Y. L. Zhu, Y. J. Wang, Y. L. Tang, Y. Liu, S. R. Zhang, J. Y. Ma, and X. L. Ma, Appl. Phys. Lett. 111, 052901 (2017).

[28] Q. Zhang, L. Xie, G. Liu, S. Prokhorenko, Y. Nahas, X. Pan, L. Bellaiche, A. Gruverman, and N. Valanoor, Adv. Mater. 29, 1702375 (2017).

[29] A. K. Yadav, C. T. Nelson, Z. H. S. L. Hsu, J. D. Clarkson, C. M. Schlepüetz, A. R. Damodaran, P. Shafer, E. Arenholz, L. R. Dedon, A. Vishwanath, A. M. Minor, L. Q. Chen, J. F. Scott, L. W. Martin, and R. Ramesh, Nature (London) 530, 198 (2016).

[30] P. Shafer, P. García-Fernández, P. Aguado-Puente, A. R. Damodaran, A. K. Yadav, C. T. Nelson, S.-L. Hsu, J. C. Wojdeł, J. Íñiguez, L. W. Martin, E. Arenholz, J. Junquera, and R. Ramesh, Proc. Natl. Acad. Sci. (USA) 115, 915 (2018).

[31] S. Das, Y. L. Tang, Z. Hong, M. A. P. Gonçalves, M. R. McCarter, C. Klewe, K. X. Nguyen, F. Gómez-Ortiz, P. Shafer, E. Arenholz, V. A. Stoica, S.-L. Hsu, B. Wang, C. Ophus, J. F. Liu, C. T. Nelson, S. Saremi, B. Prasad, A. B. Mei, D. G. Schlom, J. Íñiguez, P. García-Fernández, D. A. Muller, 
L. Q. Chen, J. Junquera, L. W. Martin, and R. Ramesh, Nature (London) 568, 368 (2019).

[32] A. M. Bratkovsky and A. P. Levanyuk, Phys. Rev. B 63, 132103 (2001).

[33] I. Luk'yanchuk, A. Sené, and V. M. Vinokur, Phys. Rev. B 98, 024107 (2018).

[34] J. Íñiguez, P. Zubko, I. Luk'yanchuk, and A. Cano, Nat. Rev. Mater. 4, 243 (2019).

[35] S. Salahuddin and S. Datta, Nano Lett. 8, 405 (2008).

[36] Y.-C. Liang, T.-B. Wu, H.-Y. Lee, and Y.-W. Hsieh, J. Appl. Phys. 96, 584 (2004).

[37] L. Qiao and X. Bi, CrystEngComm 13, 1693 (2011).

[38] Y.-T. Liu, S.-J. Chiu, H.-Y. Lee, and S.-Y. Chen, Thin Solid Films 529, 66 (2013), tACT2011 International Thin Films Conference.

[39] G. De Luca, N. Strkalj, S. Manz, C. Bouillet, M. Fiebig, and M. Trassin, Nat. Commun. 8, 1419 (2017).

[40] E.-J. Guo, M. A. Roldan, X. Sang, S. Okamoto, T. Charlton, H. Ambaye, H. N. Lee, and M. R. Fitzsimmons, Phys. Rev. Mater. 2, 114404 (2018).

[41] K. Ueda, H. Saeki, H. Tabata, and T. Kawai, Solid State Commun. 116, 221 (2000).

[42] C. v. Korff Schmising, M. Bargheer, M. Kiel, N. Zhavoronkov, M. Woerner, T. Elsaesser, I. Vrejoiu, D. Hesse, and M. Alexe, Phys. Rev. B 73, 212202 (2006).

[43] C. v. Korff Schmising, M. Bargheer, M. Kiel, N. Zhavoronkov, M. Woerner, T. Elsaesser, I. Vrejoiu, D. Hesse, and M. Alexe, Phys. Rev. Lett. 98, 257601 (2007).

[44] S. J. Callori, J. Gabel, D. Su, J. Sinsheimer, M. V. FernandezSerra, and M. Dawber, Phys. Rev. Lett. 109, 067601 (2012).

[45] J. Junquera and P. Ghosez, Nature (London) 422, 506 (2003).

[46] M. Stengel, D. Vanderbilt, and N. A. Spaldin, Nat. Mater. 8, 392 (2009).

[47] D. J. Kim, J. Y. Jo, Y. S. Kim, Y. J. Chang, J. S. Lee, J.-G. Yoon, T. K. Song, and T. W. Noh, Phys. Rev. Lett. 95, 237602 (2005).

[48] C. Lichtensteiger, M. Dawber, N. Stucki, J.-M. Triscone, J. Hoffman, J.-B. Yau, C. H. Ahn, L. Despont, and P. Aebi, Appl. Phys. Lett. 90, 052907 (2007).

[49] V. Nagarajan, J. Junquera, J. Q. He, C. L. Jia, R. Waser, K. Lee, Y. K. Kim, S. Baik, T. Zhao, R. Ramesh, P. Ghosez, and K. M. Rabe, J. Appl. Phys. 100, 051609 (2006).

[50] P. Zubko, H. Lu, C.-W. Bark, X. Martí, J. Santiso, C.-B. Eom, G. Catalan, and A. Gruverman, J. Phys.: Condens. Matter 29, 284001 (2017).

[51] N. Strkalj, G. De Luca, M. Campanini, S. Pal, J. Schaab, C. Gattinoni, N. A. Spaldin, M. D. Rossell, M. Fiebig, and M. Trassin, Phys. Rev. Lett. 123, 147601 (2019).
[52] J. J. P. Peters, G. Apachitei, R. Beanland, M. Alexe, and A. M. Sanchez, Nat. Commun. 7, 13484 (2016).

[53] S. J. Callori, Ph.D. thesis, Stony Brook University, 2013.

[54] S. J. May, A. B. Shah, S. G. E. te Velthuis, M. R. Fitzsimmons, J. M. Zuo, X. Zhai, J. N. Eckstein, S. D. Bader, and A. Bhattacharya, Phys. Rev. B 77, 174409 (2008).

[55] J. Xia, W. Siemons, G. Koster, M. R. Beasley, and A. Kapitulnik, Phys. Rev. B 79, 140407(R) (2009).

[56] R. Bouchard and J. Gillson, Mater. Res. Bull. 7, 873 (1972).

[57] X. D. Wu, S. R. Foltyn, R. C. Dye, Y. Coulter, and R. E. Muenchausen, Appl. Phys. Lett. 62, 2434 (1993).

[58] X. Shen, X. Qiu, D. Su, S. Zhou, A. Li, and D. Wu, J. Appl. Phys. 117, 015307 (2015).

[59] M. Dawber, N. Stucki, C. Lichtensteiger, S. Gariglio, P. Ghosez, and J.-M. Triscone, Adv. Mater. 19, 4153 (2007).

[60] S. C. Gausepohl, M. Lee, R. A. Rao, and C. B. Eom, Phys. Rev. B 54, 8996 (1996).

[61] S. K. Streiffer, J. A. Eastman, D. D. Fong, C. Thompson, A. Munkholm, M. V. Ramana Murty, O. Auciello, G. R. Bai, and G. B. Stephenson, Phys. Rev. Lett. 89, 067601 (2002).

[62] L. Landau and E. Lifshits, Phys. Z. Sow. 8, 153 (1935).

[63] C. Kittel, Phys. Rev. 70, 965 (1946).

[64] R. Takahashi, O. Dahl, E. Eberg, J. K. Grepstad, and T. Tybell, J. Appl. Phys. 104, 064109 (2008).

[65] D. D. Fong, A. M. Kolpak, J. A. Eastman, S. K. Streiffer, P. H. Fuoss, G. B. Stephenson, C. Thompson, D. M. Kim, K. J. Choi, C. B. Eom, I. Grinberg, and A. M. Rappe, Phys. Rev. Lett. 96, 127601 (2006).

[66] E. A. Eliseev, I. S. Vorotiahin, Y. M. Fomichov, M. D. Glinchuk, S. V. Kalinin, Y. A. Genenko, and A. N. Morozovska, Phys. Rev. B 97, 024102 (2018).

[67] S. Fernandez-Peña, C. Lichtensteiger, P. Zubko, C. Weymann, S. Gariglio, and J.-M. Triscone, APL Mater. 4, 086105 (2016).

[68] G. Catalan, D. O’Neill, R. M. Bowman, and J. M. Gregg, Appl. Phys. Lett. 77, 3078 (2000).

[69] A. Jonscher, Dielectric Relaxation in Solids (Chelsea Dielectrics Press, London, 1983).

[70] F. D. Morrison, D. J. Jung, and J. F. Scott, J. Appl. Phys. 101, 094112 (2007).

[71] Y. N. Huang, X. Li, Y. Ding, Y. N. Wang, H. M. Shen, Z. F. Zhang, C. S. Fang, S. H. Zhuo, and P. C. W. Fung, Phys. Rev. B 55, 16159 (1997).

[72] M. P. Warusawithana, E. V. Colla, J. N. Eckstein, and M. B. Weissman, Phys. Rev. Lett. 90, 036802 (2003).

[73] R. Plonka, R. Dittmann, N. A. Pertsev, E. Vasco, and R. Waser, Appl. Phys. Lett. 86, 202908 (2005). 